\title{
Apoptosis and antitumor effects induced by the combination of an mTOR inhibitor and an autophagy inhibitor in human osteosarcoma MG63 cells
}

\author{
RYOSUKE HORIE, OSAMU NAKAMURA, YOSHIKI YAMAGAMI, MASAKI MORI, \\ HIDEKI NISHIMURA, NATSUKO FUKUOKA and TETSUJI YAMAMOTO
}

Department of Orthopaedic Surgery, Kagawa University School of Medicine, Miki-cho, Kita-gun, Kagawa, Japan

Received August 4, 2015; Accepted October 12, 2015

DOI: $10.3892 /$ ijo.2015.3227

\begin{abstract}
The inhibition of the mammalian target of rapamycin (mTOR) signaling pathway promotes the initiation of autophagy. Although it remains under debate whether chemotherapy-induced autophagy in tumor cells is a protective response or is invoked to promote cell death, recent studies indicate that autophagy is a self-defense mechanism of cancer cells that are subjected to antitumor agents and that blocking autophagy can trigger apoptosis. The aim of this study was to examine the effects of rapamycin, an mTOR inhibitor, on MG63 osteosarcoma cells. We further examined whether the combination of rapamycin and the small molecule inhibitor of autophagy Spautin-1 (specific and potent autophagy inhibitor-1) enhanced the rapamycin-induced apoptosis in MG63 cells. We examined the effects of rapamycin treatment on cell proliferation, phosphorylation of mTOR pathway components, and autophagy by western blot analysis. Furthermore, we examined the effects of rapamycin with or without Spautin-1 on the induction of apoptosis by western blot analysis and immunohistochemical staining. We found that rapamycin inhibited cell proliferation and decreased the phosphorylation of mTOR pathway components in MG63 cells. Rapamycin induced the apoptosis of MG63 cells, and this apoptosis was enhanced by Spautin-1. It was considered that Spautin-1 suppressed the protective mechanism induced by rapamycin in tumor cells and induced apoptosis. Therefore, the combination of an mTOR inhibitor and an autophagy inhibitor may be effective in the treatment of osteosarcoma because it effectively induces the apoptotic pathway.
\end{abstract}

\section{Introduction}

Osteosarcoma derives from primitive bone-forming mesenchymal cells and is the most common primary bone malignancy.

Correspondence to: Dr Osamu Nakamura, Department of Orthopaedic Surgery, Kagawa University, 1750-1 Ikenobe, Miki-cho, Kita-gun, Kagawa 761-0793, Japan

E-mail: osamunak@med.kagawa-u.ac.jp

Key words: autophagy, apoptosis, mTOR, Spautin-1, osteosarcoma
The incidence (and 95\% confidence intervals) of osteosarcoma for all races and both sexes is $4.0 \%$ (3.5-4.6) among those aged 0-14 years and 5.0\% (4.6-5.6) among those aged 0-19 years per year (1). Therapy for osteosarcoma has made good progress with the development of surgery and screening technologies and with the combination of neoadjuvant chemotherapy. However, the problems of metastasis, recurrence and chemoresistance have yet to be solved. In recent years, drugs that target specific molecules have been developed as treatments for human malignancies (2). These drugs often selectively inhibit specific molecules, such as growth factor receptors or intracellular signaling proteins that are related to tumor proliferation, migration and/or metastasis (3).

Mammalian target of rapamycin (mTOR) is an essential serine/threonine kinase that belongs to the phosphoinositide3-OH kinase (PI3K)-related kinase family (4). It regulates multiple cellular processes, including survival, cell growth, proliferation, migration and angiogenesis, in many kinds of cancers $(5,6)$. The inhibition of mTOR affects mTOR pathwaymediated cellular transcription and translation, leading to cell cycle arrest and anti-angiogenesis. Regarding the antitumor mechanisms of mTOR inhibitors, several studies have indicated that inhibitors of Akt and its downstream target, mTOR signaling, have anticancer effects as the inhibition of the mTOR pathway contributes to the initiation of autophagy (7-9).

mTOR nucleates two complexes: mTOR complex 1 (mTORC1) and mTOR complex 2 (mTORC2). These complexes are key and essential regulators of cellular pathways that control the initiation of mRNA translation and ribosome biogenesis and exhibit important monitoring effects on cell metabolism, lipolysis and autophagy (10-12). Autophagy is activated during starvation to provide an alternative energy source through self-digestion. Thus, autophagy serves as a temporary survival mechanism. Autophagy is also important in the induction of tumor cell death (13) and excessive autophagy triggers autophagic cell death in tumors $(14,15)$. However, it remains under debate whether chemotherapy-induced autophagy in tumor cells is a protective response or is invoked to promote cell death (16). Not surprisingly, there is an intricate relationship between autophagy and apoptosis. Recent studies indicate that autophagy can function as a self-defense mechanism in 
cells that are exposed to antitumor agents, and that blocking autophagy can trigger the activation of apoptosis (17-19).

Recently, a potent small molecule inhibitor of autophagy named Spautin-1 (specific and potent autophagy inhibitor-1) was discovered. This molecule promotes the degradation of Vps34- $\mathrm{PI}_{3}$ kinase complexes by inhibiting two ubiquitinspecific peptidases, USP10 and USP13, that target the Beclin-1 subunit of Vps34 complexes. USP10 mediates the deubiquitination of $\mathrm{p} 53$, and as Beclin-1 regulates the deubiquitination activity of USP10 and USP13, Beclin-1 can control the levels of p53. Through this mechanism, Spautin-1 increases cancer cell death under the condition of nutrient deprivation when autophagy would normally act as a survival mechanism in the metabolically stressed cells (20).

The aim of this study was to examine the effects of the mTOR inhibitor rapamycin on osteosarcoma cells. We investigated whether rapamycin modulated the phosphorylation of proteins in the Akt/mTOR signaling pathway and/or induced autophagy in osteosarcoma cells. In addition, we hypothesized that the combination of rapamycin and Spautin-1 would induce strong antitumor effects in osteosarcoma cells by blocking self-defensive autophagy.

\section{Materials and methods}

Chemical reagents. Rapamycin (CCI-779) and the autophagy inhibitor, Spautin-1, were purchased from Calbiochem (San Diego, CA, USA). Both reagents were dissolved in dimethyl sulfoxide (DMSO) and rapamycin was stored at $-20^{\circ} \mathrm{C}$ and Spautin-1 was stored at $8^{\circ} \mathrm{C}$. Chloroquine diphosphate salt (CQ) was purchased from Sigma-Aldrich (St. Louis, MO, USA) and dissolved in distilled water to a concentration of $100 \mathrm{mM}$, and was stored at room temperature.

Cell lines and cell culture. The MG63 cell line, derived from an osteogenic sarcoma, was used in this study. The cells were grown in Dulbecco's modified Eagle's medium (DMEM; Sigma-Aldrich) containing $10 \%$ fetal bovine serum (FBS; Sigma-Aldrich) and $100 \mathrm{U} / \mathrm{ml}$ penicillin, and were routinely maintained at $37^{\circ} \mathrm{C}$ in a humidified $5 \% \quad \mathrm{CO}_{2}$ atmosphere. Cultures at the mid-log phase were used for the experiments.

For the detection of autophagy and the apoptosis assay, cells were divided into four groups: control (no inhibitor), Rap (rapamycin), Spa (Spautin-1), and Rap-plus-Spa (rapamycin + Spautin-1) groups.

In vitro proliferation assay. Cell proliferation was determined by the CellTiter $96^{\circledR}$ AQueous One Solution Cell Proliferation assay (Promega Corp., Madison, WI, USA). Briefly, cells were trypsinized and seeded at a density of $\sim 1 \times 10^{4}$ cells/well in 96-well cell culture plates containing $200 \mu \mathrm{l} /$ well of culture medium with $10 \%$ FBS. After $48 \mathrm{~h}$, the medium was replaced with medium containing $10 \% \mathrm{FBS}$ and supplemented with rapamycin at a concentration of $0,0.4$, 2,10 , or $50 \mu \mathrm{M}$. After 24 or $48 \mathrm{~h}$, the medium was replaced with fresh medium containing 3-(4,5-dimethylthiazol-2-yl)5-(3-carboxymethoxyphenyl)-2-(4-sulfophenyl)-2H-tetrazolium (MTS) reagent (100 $\mu \mathrm{l}$ of medium plus $20 \mu \mathrm{l}$ of MTS regent/ well). In the experiments testing the combined effect of rapamycin and Spautin-1, cells were treated with $20 \mu \mathrm{M}$ rapamycin and $100 \mu \mathrm{M}$ Spautin-1 for $24 \mathrm{~h}$. In the experiments testing the effect of rapamycin or Spautin-1 alone, cells were treated with either $20 \mu \mathrm{M}$ rapamycin or $100 \mu \mathrm{M}$ Spautin-1 for $24 \mathrm{~h}$. The optical density of the samples was measured at $490 \mathrm{~nm}$ using an automatic microplate reader (SpectraMax ${ }^{\circledR}$ Plus384 microplate reader; Molecular Devices, Sunnyvale, CA, USA) after $2 \mathrm{~h}$ of further incubation with MTS reagent. Absorbance was directly proportional to the number of living cells. The percent viability of the cells in each well was calculated. At least three independent experiments were performed for each condition.

Western blot analyses. Cells were trypsinized and seeded at a density of $\sim 6 \times 10^{5}$ cells/well in 6 -well cell culture plates with $2 \mathrm{ml} /$ well of culture medium containing 10\% FBS. After $48 \mathrm{~h}$, cells were treated with medium containing $10 \%$ FBS and rapamycin at a concentration of $0,0.4,2,10$, or $50 \mu \mathrm{M}$ for $24 \mathrm{~h}$. In the experiments testing the combined effect of rapamycin and Spautin-1, the cells were treated with $20 \mu \mathrm{M}$ rapamycin and $100 \mu \mathrm{M}$ Spautin-1 for $24 \mathrm{~h}$. In the experiments testing the effect of rapamycin or Spautin-1 alone, the cells were treated with either $20 \mu \mathrm{M}$ rapamycin or $100 \mu \mathrm{M}$ Spautin-1 for $24 \mathrm{~h}$. Following treatment, the culture medium was replaced with lysis buffer (Cell Signaling Technology, Beverly, MA, USA) and the cells were lysed on ice for $20 \mathrm{~min}$. The cell lysates were spun at 15,000 x g using a Tabletop Micro Refrigerated Centrifuge 3500 (Kubota Shoji Co., Ltd., Tokyo, Japan) at $4^{\circ} \mathrm{C}$ for $30 \mathrm{~min}$. The supernatant was then collected as the total cellular protein extract. The protein concentrations were determined using the Protein Assay Bicinchoninate kit (Nacalai Tesque, Inc., Kyoto, Japan) and standardized using bovine serum albumin. The total cellular protein samples were loaded onto an SDS polyacrylamide gel (10 or $12.5 \%$ commercial precast gels; Wako, Tokyo, Japan), and the proteins were separated by SDS-PAGE under reducing conditions. The separated proteins were electrophoretically transferred onto nitrocellulose membranes (GE Healthcare Bio-Sciences, Piscataway, NJ, USA). The membranes were blocked for $90 \mathrm{~min}$ in blocking buffer that contained Trisbuffered saline (TBS-T) and EzBlock Chemi (Atto Co., Tokyo, Japan). They were then incubated overnight at $4^{\circ} \mathrm{C}$ with the appropriate primary antibody (Table I) diluted in blocking buffer. The specific horseradish peroxidase-conjugated secondary antibody incubation was performed overnight at $4^{\circ} \mathrm{C}$ with gentle agitation. Bound secondary antibodies were detected using the ECL Plus Western Blotting Detection system (GE Healthcare Bio-Sciences) and an LAS-1000 Plus Image Analyzer (Fujifilm Co., Tokyo, Japan). Specific signals were quantified by densitometric analysis using NIH ImageJ software.

Immunocytochemical staining for LC3. Cells were trypsinized and seeded at a density of $\sim 1 \times 10^{6}$ cells/well on $25-\mathrm{mm}$ circular coverslips (Matsunami Glass Industries, Ltd., Osaka, Japan) in $2 \mathrm{ml}$ of culture medium containing 10\% FBS overnight. In the experiments testing the effect of rapamycin, cells were treated with $20 \mu \mathrm{M}$ rapamycin for $24 \mathrm{~h}$. Cells were then fixed in $4 \%$ paraformaldehyde in phosphate buffer for $30 \mathrm{~min}$ at room temperature and washed in phosphate-buffered saline (PBS). 
Table I. Primary antibodies used in western blot analysis.

\begin{tabular}{lllll}
\hline Target & \multicolumn{1}{c}{ Source } & Host & Dilution & Second antibody \\
\hline LC-3 & MBL & Rabbit & $1: 1,000$ & Anti-rabbit \\
p62/SQSTM1 & MBL & Rabbit & $1: 1,000$ & Anti-rabbit \\
4E-BP1 & Cell Signaling & Rabbit & $1: 1,000$ & Anti-rabbit \\
phospho-4E-BP1 & Cell Signaling & Rabbit & $1: 1,000$ & Anti-rabbit \\
cleaved PARP & Cell Signaling & Rabbit & $1: 1,000$ & Anti-rabbit \\
$\alpha$-tubulin & Sigma & Mouse & $1: 1,000$ & Anti-mouse \\
\hline
\end{tabular}

For the detection of autophagy, cells were incubated with anti-LC3 antibody (code no. PM036, MBL, Nagoya, Japan) for $1 \mathrm{~h}$ at room temperature. Cells were then washed two times with PBS, incubated with anti-IgG secondary antibody (Alexa Fluor $^{\circledR}$ 488, code no. A11008; Invitrogen, Carlsbad, CA, USA) for $30 \mathrm{~min}$ at room temperature and examined under an epifluorescence microscope (FSX100, Olympus Optical Co., Ltd., Tokyo, Japan) with a 50x objective lens.

Quantification of GFP-LC3 puncta. The GFP-LC3 puncta was quantified for the detection of autophagy. MG63 cells were transfected with GFP-LC3 (P36235, Invitrogen) for $24 \mathrm{~h}$ and a CQ assay was used to determine the dynamic turnover of GFP-LC3 in autolysosomes. Because CQ is a lysosome inhibitor, GFP-LC3 puncta will increase even CQ alone. However, if we could prove that GFP-LC3 puncta is increased in the Rap group treated with CQ compared to CQ alone, it would mean autophagy induced by rapamycin is not caused by the lysosome inhibitor, therefore we used CQ assay. The transfected cells were pre-treated with $50 \mu \mathrm{M}$ CQ for $12 \mathrm{~h}$. They were then washed with PBS two times and treated with or without $20 \mu \mathrm{M}$ rapamycin for $24 \mathrm{~h}$. Next, cells were fixed with $4 \%$ paraformaldehyde for $30 \mathrm{~min}$. Images of individual GFP-LC3-expressing cells were taken under an epifluorescence microscope (FSX100, Olympus Optical Co.) with a 50x objective lens.

Fluorescence microscopy imaging of the Annexin V/propidium iodide (PI) and Hoechst 33342 triple-staining assay. Cells were trypsinized and seeded at a density of $\sim 1 \times 10^{6}$ cells/well on 25-mm circular coverslips in $2 \mathrm{ml}$ of culture medium containing 10\% FBS for $24 \mathrm{~h}$. Next, cells were washed with PBS and treated with $20 \mu \mathrm{M}$ rapamycin and/ or $100 \mu \mathrm{M}$ Spautin-1 for $24 \mathrm{~h}$. After treatment, cells were incubated for $15 \mathrm{~min}$ in a dark room with Annexin V-FITC, PI and Hoechst 33342 using the Promokine Apoptotic/ Necrotic/Healthy Cells Detection kit (PromoCell GmbH, Heidelberg, Germany). Cells were then examined under an epifluorescence microscope (FSX100, Olympus Optical Co.) with a 50x objective lens.

In vivo xenograft studies. Four-week-old female athymic BALB/c nude mice (Clea Japan, Inc., Tokyo, Japan) were maintained in pathogen-free conditions and in accordance with institutional principals. MG63 cells $\left(3.0 \times 10^{6}\right.$ cells in $0.5 \mathrm{ml}$ of medium) were injected subcutaneously into the dorsal area of mice. For this study on antitumor activity, rapamycin and
Spautin-1 were purchased and used to determine whether rapamycin and/or Spautin-1 affect tumor volume. Twenty-four mice were randomly divided into four groups: a rapamycin group (Rap; $n=6$ ), a Spautin-1 group (Spa; $n=6$ ), a combination of rapamycin and Spautin-1 group (Rap-plus-Spa; $n=6$ ) and a control group (control; $n=6$ ). After allowing 14 days for implantation, intraperitoneal injections of the drugs were started. Throughout the experimental period, the mice were slowly injected intraperitoneally with $0.2 \mathrm{ml}$ of the following five times a week for 4 weeks: $8 \mathrm{mg} / \mathrm{kg}$ of rapamycin for the Rap group; $40 \mathrm{mg} / \mathrm{kg}$ of Spautin-1 for the Spa group; $8 \mathrm{mg} / \mathrm{kg}$ of rapamycin plus $40 \mathrm{mg} / \mathrm{kg}$ of Spautin-1 for the Rap-plus-Spa group; and PBS only for the control group. After implantation, the tumor dimensions were measured once a week. Tumor volume was calculated according to the formula $\mathrm{V}=\pi / 6 \mathrm{x}$ $a^{2} x b$, where $a$ and $b$ represent the shortest and the longest dimensions of the tumor. All in vivo studies were performed in accordance with The Guide for the Care and Use of Laboratory Animals (Washington, DC: National Academy Press, 1996) and approved by the Institutional Animal Care and Use Committee of our institution.

Statistical analysis. Statistical analyses for the cell proliferation assay were performed using GraphPad Prism 5 software (GraphPad, San Diego, CA, USA) with one- or two-way ANOVA followed by post hoc analysis. A value of $p<0.05$ was considered to indicate a statistically significant difference.

\section{Results}

Rapamycin inhibits the proliferation of MG63 cells. First, we assessed the effects of rapamycin on cellular proliferation using the CellTiter 96R AQueous One Solution Cell Proliferation assay. MG63 cells were cultured in the presence of increasing doses of rapamycin for 24 or $48 \mathrm{~h}$. As shown in Fig. 1, rapamycin inhibited MG63 proliferation in a dose- and time-dependent manner. The $\mathrm{IC}_{50}$ value of rapamycin at $24 \mathrm{~h}$ was $19.36 \mu \mathrm{M}$.

Rapamycin-induced MG63 cell death is enhanced by Spautin-1. We then examined the effects of rapamycin and/or Spautin-1 on MG63 cell proliferation. Based on the $24-\mathrm{h} \mathrm{IC}_{50}$ of rapamycin, we examined the proliferation of MG63 cells treated for $24 \mathrm{~h}$ with $20 \mu \mathrm{M}$ rapamycin, $100 \mu \mathrm{M}$ Spautin-1, or $20 \mu \mathrm{M}$ rapamycin and $100 \mu \mathrm{M}$ Spautin-1. Cell proliferation was significantly lower in the Rap-plus-Spa group than in the Rap group (p<0.05) (Fig. 2). 


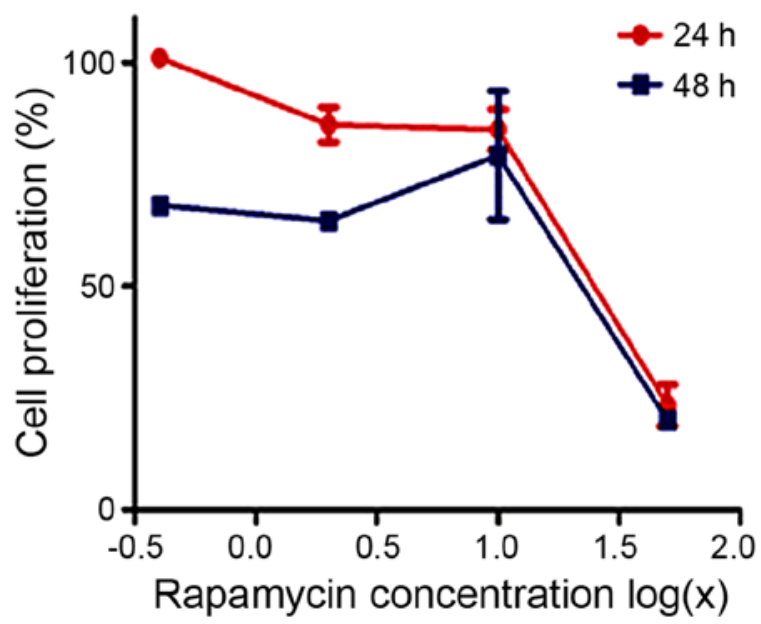

Figure 1. Cell proliferation assay was used to investigate the effects of rapamycin on the proliferation of cultured MG63 cells. Rapamycin inhibited MG63 cell proliferation in a dose- and time-dependent manner.

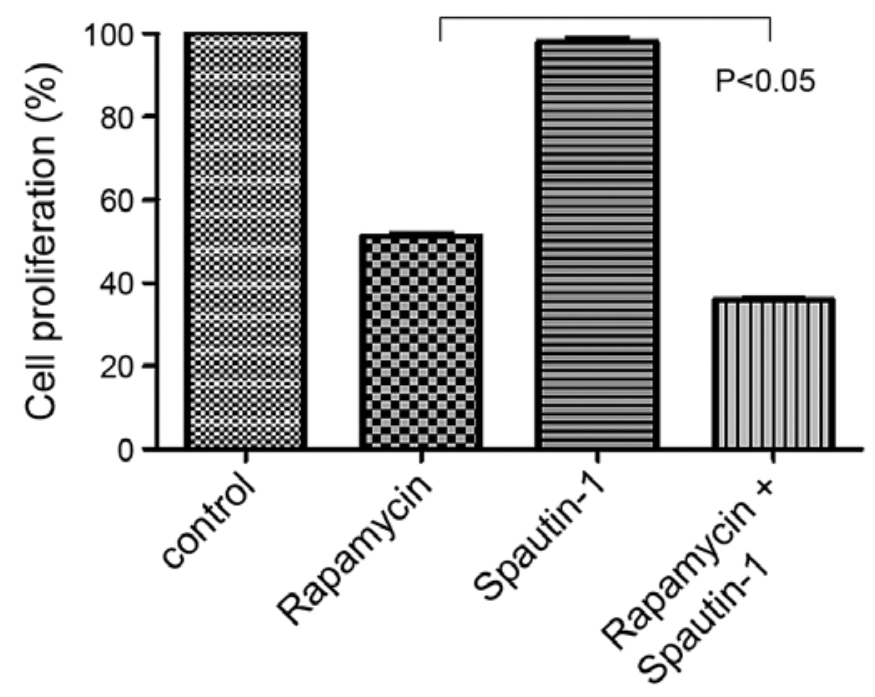

Figure 2. MG63 cell proliferation was lower in the rapamycin and Spautin-1treated cells than in the rapamycin-treated cells $(\mathrm{p}<0.05)$.

Western blot analysis. Western blot analysis demonstrated that treatment with rapamycin induced the phosphorylation of 4E-binding protein (4E-BP1), one of the key components in the mTOR pathway. Additionally, we examined the expression of the autophagy-related gene complex, p62/SQSTM1, and LC-3 in MG63 cells exposed to various concentrations of rapamycin (ranging from 0.4 to $50 \mu \mathrm{M}$ ) for $24 \mathrm{~h}$ (Fig. 3A). Treatment with rapamycin resulted in a dose-dependent decrease in the levels of phospho-4E-BP1, which is a downstream effector of mTOR. These findings indicate that rapamycin affected the mTOR pathway by inhibiting the phosphorylation of downstream effectors of mTOR. LC-3II expression was used as an autophagic marker. The p62 protein, also called sequestosome 1 (SQSTM1), is commonly found in inclusion bodies containing polyubiquitinated protein aggregates, which are degraded by autophagy (21). Treatment with rapamycin resulted in a dose-dependent increase in the expression of LC-3II in the MG63 cells. In contrast, p62/SQSTM1 expression decreased

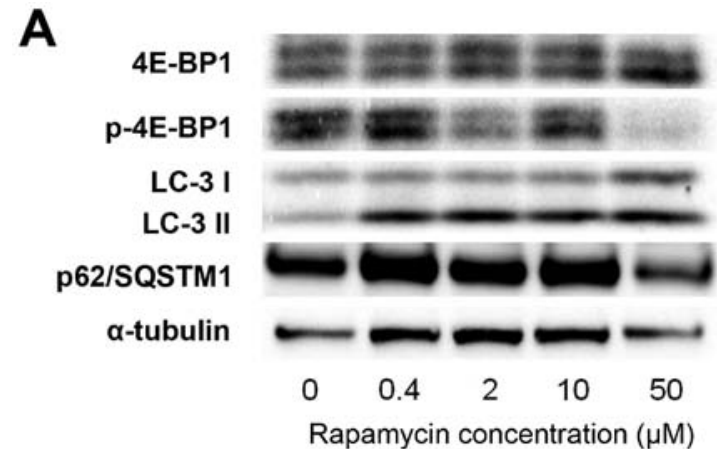

B

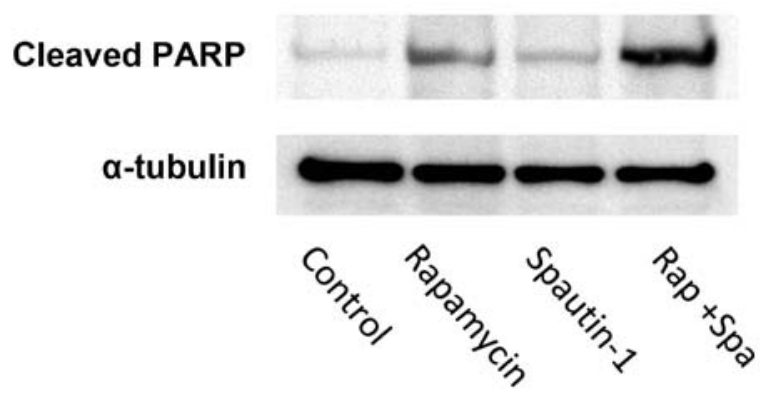

Figure 3. Western blot analysis to investigate the effects of rapamycin on components of the mTOR pathway. (A) Phospho-4E-BP1 expression levels were decreased in a dose-dependent manner following treatment with rapamycin. Treatment with rapamycin resulted in a dose-dependent increase in LC-3II and a decrease in p62/SQSTM1 expression. (B) Analysis of the effects of treatment with rapamycin and/or Spautin-1. The production of cleaved PARP was higher in the Rap-plus-Spa group than in the Rap and Spa groups.

in a dose-dependent manner (Fig. 3A). In cells treated with rapamycin, the production of cleaved PARP slightly increased. On the other hand, in cells treated with rapamycin plus Spautin-1, the production of cleaved PARP strongly increased (Fig. 3B).

Immunocytochemistry of LC3 for the detection of autophagy. Immunochemical staining for LC3 was performed on MG63 cells. There was a strong increase in LC3-positive puncta (autophagosomes) in the Rap group (Fig. 4).

Quantification of GFP-LC3 puncta. Similar to the immunocytochemical staining assay of LC3, GFP-LC3-positive puncta were slightly increased in the Rap group and were significantly increased in the Rap group treated with CQ (Fig. 5).

Fluorescence microscopy images of the Annexin V/PI and Hoechst 33342 triple-staining assay for the detection of apoptosis. We next used an Annexin V/PI and Hoechst 33342 triple-staining assay to detect apoptotic cells. Hoechst 33342 (blue) is a marker for live cells, Annexin V-FITC (green) is a marker for early apoptosis, and PI (red) is a marker for late apoptosis and necrosis. We observed several Annexin V-FITCpositive cells (early stage of apoptosis) and a high number of Annexin V-FITC plus PI-positive cells (late stage of apoptosis) in the Rap-plus-Spa group (Fig. 6).

Effect of rapamycin and Spautin-1 on tumor growth in xenograft models. The antitumor activities of rapamycin and 


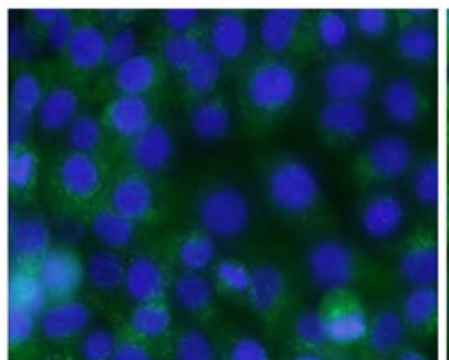

Control

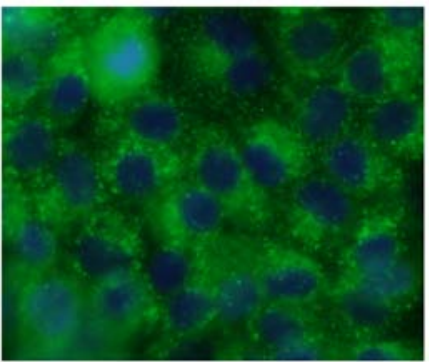

Rapamycin

Figure 4. Rapamycin induces autophagy in MG63 cells. Compared with the control group, there were more LC3-positive puncta (autophagosomes) in the Rap group.

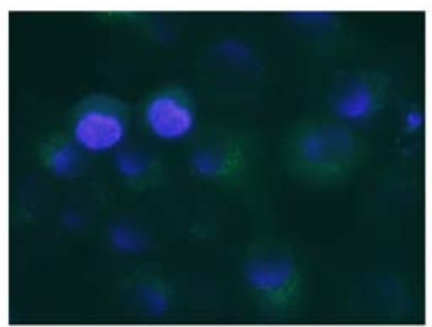

Control

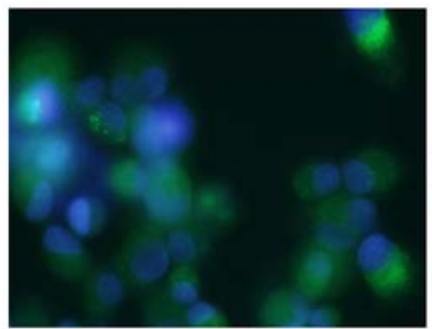

$\mathrm{CQ}$

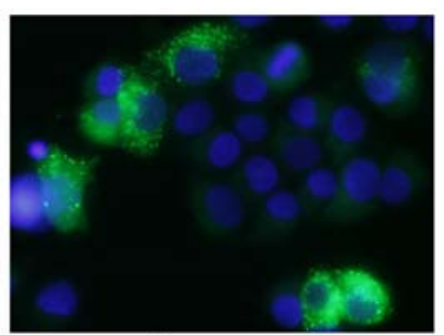

Rapamycin

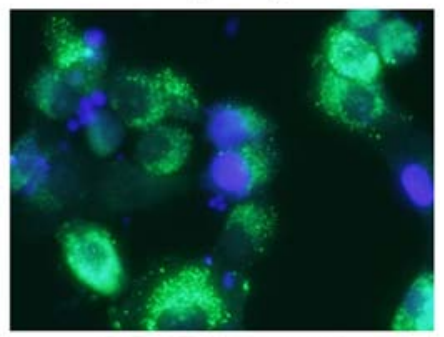

CQ+Rapamycin

Figure 5. Similar to the results of the immunocytochemical staining assay of LC3, a larger number of GFP-LC3-positive puncta was seen in the Rap group when compared with the control group.

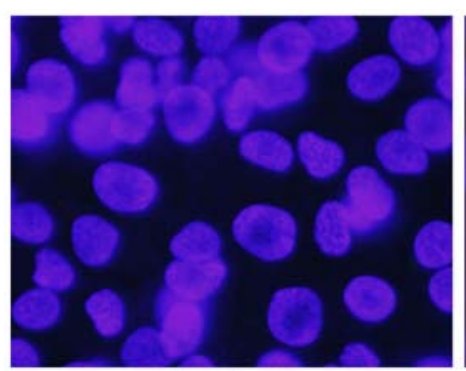

Control

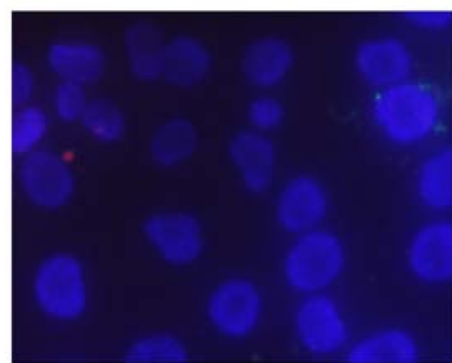

Spautin-1

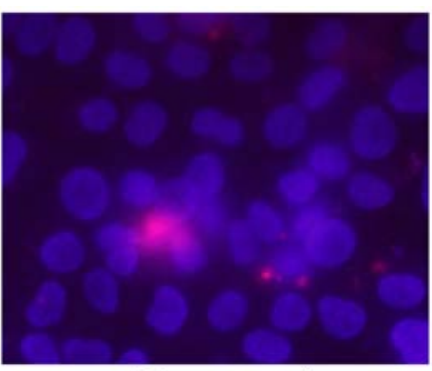

Rapamycin

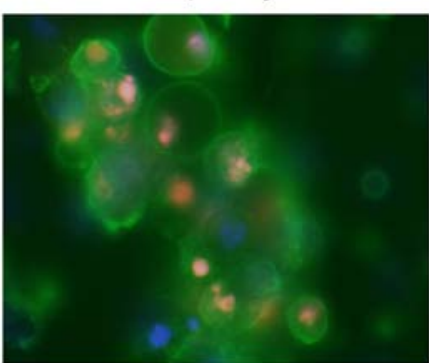

Rapamycin + Spautin-1

Figure 6. Fluorescent staining for the detection of apoptosis. Apoptotic cells were detected by an Annexin V/propidium iodide (PI) and Hoechst 33342 triplestaining assay. Cells were stained by blue (Hoechst 33342), green (Annexin V) and red (PI). There were more Annexin V-stained cells in the group treated with both rapamycin and Spautin-1 than in the other groups. Rap, rapamycin; Spa, Spautin-1. 

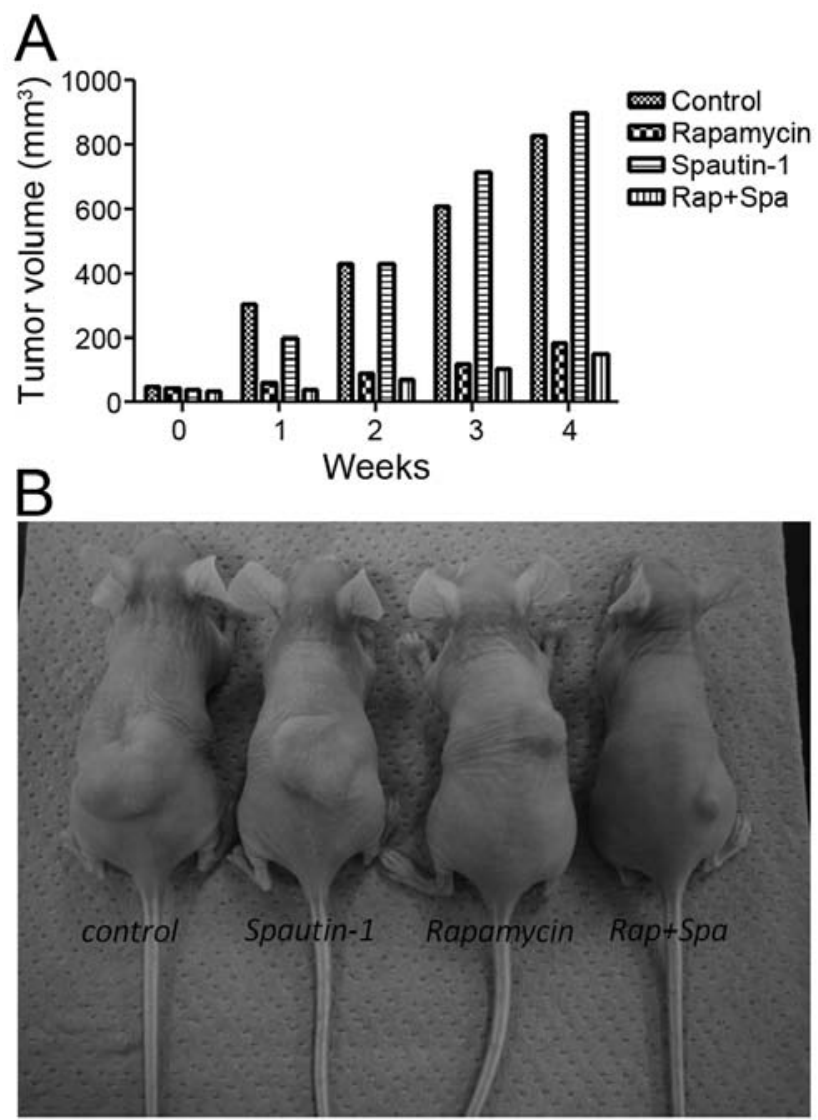

Figure 7. Tumorigenesis of MG63 xenograft models. (A) Tumor growth in the Rap group and the Rap-plus-Spa group was significantly more inhibited than that in the control and Spa groups. (B) At the end of the experiment, the mean tumor volume in the Rap-plus-Spa group was smaller than that of the Rap group, but there was no significant difference.

Spautin-1 in nude mice bearing MG63 xenografts was investigated. Implantation of $3.0 \times 10^{6}$ cells into the dorsal area of nude mice resulted in the development of tumors in $100 \%$ of the animals. After 1 week, tumor growth in the Rap group and the Rap-plus-Spa group was significantly inhibited when compared with that in the control group and the Spa group. At the end of the experimental period, the mean tumor volume of the Rap group and the Rap-plus-Spa group was 181 and $146 \mathrm{~mm}^{3}$, respectively (Fig. 7B). Although there was no significant difference between the Rap group and the Rap-plus-Spa group, tumor growth in the Rap-plus-Spa group was less than that seen in the Rap group (Fig. 7A). Throughout the experimental period, no side effects, such as body weight loss, were observed in the treatment groups.

\section{Discussion}

mTOR signaling pathway. mTOR is a serine/threonine kinase with a catalytic subunit of two biochemically distinct complexes called mTORC1 and mTORC2. mTORC1 contains Raptor, mLST8 and PRAS40, while mTORC2 contains mTOR, Rictor, mSIN1 and Protor-1. mTORC1 senses several cellular/environmental signals, including protein misfolding, nutrient availability and growth signals. mTORC1 controls cell growth through the phosphorylation of S6K and 4E-BP1, and regulates autophagy; in contrast, mTORC2 is not a direct regulator of autophagy. Rapamycin, which is a specific inhibitor of mTORC1, has been shown to selectively and completely block mTORC1-dependent p70S6K phosphorylation and partially block 4E-BP1 phosphorylation (22). In this study, we observed that rapamycin suppressed the phosphorylation of 4E-BP1 in a dose-dependent manner. This result demonstrated that the mTOR pathway is a target of rapamycin in MG63 cells, as previously shown in several reports.

Apoptosis induced by rapamycin is enhanced by an autophagy inhibitor. Autophagy is a process in which subcellular membranes undergo dynamic morphological changes (autophagosomes form and fuse with lysosomes) leading to the degradation of cellular proteins and cytoplasmic organelles. Autophagy plays a protective role when cells encounter environmental stress such as starvation or pathogen infection $(23,24)$. Autophagy also occurs under pathological conditions, such as neurodegenerative diseases or hereditary myopathies (25). Recent accumulating evidence indicates that autophagy often plays a role in malignant diseases. Specifically, autophagy is believed to play an important role in tumor development. During the early stages of tumor formation, autophagy functions as a tumor suppressor, and autophagic activity is often impaired in cancer cells. However, autophagy has also been shown to play a role as a self-defense mechanism in promoting tumor cell resistance to chemotherapy (26). Some anticancer drugs that lead to apoptosis can also induce autophagy-related cell death in cancer cell lines $(27,28)$. In this study, we demonstrated that apoptosis induced by an mTOR inhibitor was enhanced by the addition of an autophagy inhibitor. As such, apoptosis is considered to be closely related to autophagy-related cell death.

Autophagy is induced by an mTOR inhibitor. In this study, our western blot results revealed that rapamycin treatment suppressed the phosphorylation of 4E-BP1 and induced a dose-dependent upregulation of the expression of the autophagy marker LC3-II and downregulation of p62/SQSTM1 in MG63 cells. In addition, immunocytochemical analysis by the staining of LC3 was performed. In rapamycin-treated cells, the number of LC3-positive puncta was markedly increased. Similar to the results of the immunocytochemical staining assay for LC3, the number of GFP-LC3 puncta was slightly increased in the Rap group and was significantly increased in the Rap group treated with CQ. These results indicate that suppression of the mTOR pathway is involved in the induction of autophagy. Furthermore, GFP-LC3-transfected cells treated with a combination of CQ and rapamycin demonstrated activation of the autophagy pathway. Our previous study also demonstrated that rapamycin can induce cytoprotective autophagy in human malignant fibrous histiocytoma (MFH) cell lines by activating the MEK/ERK signaling pathway, and that the rapamycin-induced apoptosis can be enhanced by a MEK inhibitor that was used as an autophagy inhibitor (29).

Autophagy inhibitor Spautin-1. Spautin-1 is an inhibitor of autophagy. It promotes the degradation of Vps34 complexes by inhibiting USP10 and USP13, two ubiquitin-specific 
peptidases that target the deubiquitination of Beclin-1. Several reports have reported the use of Spautin-1 as an autophagy inhibitor for the treatment of malignancy. For example, Correa et al reported that a combination of AKT inhibition and autophagy blockage would prove efficacious to reduce residual epithelial ovarian cancer cells that cause ovarian cancer recurrence (30). However, there have been no reports on the use of Spautin-1 for the treatment of osteosarcoma. Thus, to the best of our knowledge, this is the first report of the use of Spautin-1 for the treatment of osteosarcoma.

Combination therapy with rapamycin and Spautin-1. Recent reports indicate that when an autophagy inhibitor, such as 3-methyladenine (3-MA), is combined with chemotherapeutic drugs, it triggers apoptosis in some cancer cells (31). Our previous study demonstrated that the combination of temsirolimus (CCI-779, an analog of rapamycin) and 3-MA suppressed autophagy and induced apoptosis in MFH cell lines (32). In this study, cell proliferation was significantly lower in the Rap-plus-Spa group than in the Rap group, indicating that Spautin-1 enhanced the rapamycin-mediated suppression of osteosarcoma cell proliferation. In addition, we demonstrated by western blot analysis that the production of cleaved PARP strongly increased in the Rap-plus-Spa group, and by immunocytochemical analysis, that the number of Annexin V-positive cells markedly increased in the Rap-plus-Spa group. Furthermore, in vivo experiments demonstrated that tumor growth was inhibited in the Rap-plus-Spa group when compared with that in the Rap group despite no significant difference in body weight between the two groups. Autophagy appears to function as a protective mechanism in rapamycin-treated MG63 cells and blocking autophagy using Spautin-1 can promote the activation of apoptosis. These results suggest that the combination of rapamycin and Spautin-1 can potently induce apoptotic cell death in MG63 cells with the inhibition of autophagy.

In this study, only MG63 cell line was examined. Furthermore, in vivo experiment, the autophagic and apoptotic activities were not proved in treated mouse xenografts. Of course, further experiments are needed for these limitations. For example, other osteosarcoma cell lines should be used, and immunostaining assays for detection of autophagy and apoptosis should be analyzed in excised tumor models in vivo.

In conclusion, this study demonstrated that rapamycin induced autophagy in MG63 cells by inhibiting phosphorylation of mTOR pathway components, and that rapamycin-induced apoptosis was enhanced by Spautin-1. These results suggest that self-protective mechanisms involving mTOR inhibitors are hindered by the inhibition of autophagy in MG63 cells. Therefore, the combination of an mTOR inhibitor (e.g., rapamycin) and an autophagy inhibitor (e.g., Spautin-1) may offer an effective treatment for osteosarcoma as this combination effectively activates apoptotic pathways.

\section{Acknowledgements}

The authors thank Mr. Toshitaka Nakagawa (Division of Research Instrument and Equipment, Kagawa University School of Medicine, Kagawa, Japan) for providing technical assistance with epifluorescence microscopy.

\section{References}

1. Ottaviani G and Jaffe N: The epidemiology of osteosarcoma. Cancer Treat Res 152: 3-13, 2009.

2. Wardelmann E, Chemnitz JM and Wendtner CM: Targeted therapy of soft tissue sarcomas. Onkologie 35 (Suppl 1): 21-27, 2012.

3. Arslan MA, Kutuk O and Basaga H: Protein kinases as drug targets in cancer. Curr Cancer Drug Targets 6: 623-634, 2006.

4. Hay N and Sonenberg N: Upstream and downstream of mTOR. Genes Dev 18: 1926-1945, 2004.

5. Gridelli C, Maione P and Rossi A: The potential role of mTOR inhibitors in non-small cell lung cancer. Oncologist 13: 139-147, 2008.

6. Mita MM and Tolcher AW: The role of mTOR inhibitors for treatment of sarcomas. Curr Oncol Rep 9: 316-322, 2007.

7. Kim KW, Mutter RW, Cao C, Albert JM, Freeman M, Hallahan DE and Lu B: Autophagy for cancer therapy through inhibition of pro-apoptotic proteins and mammalian target of rapamycin signaling. J Biol Chem 281: 36883-36890, 2006.

8. Hung JY, Hsu YL, Li CT, Ko YC, Ni WC, Huang MS and Kuo PL: 6-Shogaol, an active constituent of dietary ginger, induces autophagy by inhibiting the AKT/mTOR pathway in human non-small cell lung cancer A549 cells. J Agric Food Chem 57: 9809-9816, 2009.

9. Tan ML, Ooi JP, Ismail N, Moad AI and Muhammad TS: Programmed cell death pathways and current antitumor targets. Pharm Res 26: 1547-1560, 2009.

10. Laplante M and Sabatini DM: mTOR signaling in growth control and disease. Cell 149: 274-293, 2012.

11. Zoncu R, Efeyan A and Sabatini DM: mTOR: From growth signal integration to cancer, diabetes and ageing. Nat Rev Mol Cell Biol 12: 21-35, 2011.

12. Beauchamp EM and Platanias LC: The evolution of the TOR pathway and its role in cancer. Oncogene 32: 3923-3932, 2013.

13. Gozuacik D and Kimchi A: Autophagy as a cell death and tumor suppressor mechanism. Oncogene 23: 2891-2906, 2004.

14. Roca H, Varsos Z and Pienta KJ: CCL2 protects prostate cancer PC3 cells from autophagic death via phosphatidylinositol 3-kinase/AKT-dependent survivin up-regulation. J Biol Chem 283: 25057-25073, 2008.

15. Rami A and Kögel D: Apoptosis meets autophagy-like cell death in the ischemic penumbra: Two sides of the same coin? Autophagy 4: 422-426, 2008.

16. Kondo Y, Kanzawa T, Sawaya $R$ and Kondo S: The role of autophagy in cancer development and response to therapy. Nat Rev Cancer 5: 726-734, 2005.

17. Liu D, Yang Y, Liu Q and Wang J: Inhibition of autophagy by 3-MA potentiates cisplatin-induced apoptosis in esophageal squamous cell carcinoma cells. Med Oncol 28: 105-111, 2011.

18. Kanematsu S, Uehara N, Miki H, Yoshizawa K, Kawanaka A, Yuri T and Tsubura A: Autophagy inhibition enhances sulforaphane-induced apoptosis in human breast cancer cells. Anticancer Res 30: 3381-3390, 2010.

19. Ren Y, Huang F, Liu Y, Yang Y, Jiang Q and Xu C: Autophagy inhibition through PI3K/Akt increases apoptosis by sodium selenite in NB4 cells. BMB Rep 42: 599-604, 2009.

20. Liu J, Xia H, Kim M, Xu L, Li Y, Zhang L, Cai Y, Norberg HV, Zhang T, Furuya T, et al: Beclin1 controls the levels of p53 by regulating the deubiquitination activity of USP10 and USP13. Cell 147: 223-234, 2011.

21. Pankiv S, Clausen TH, Lamark T, Brech A, Bruun JA, Outzen H, Øvervatn A, Bjørkøy G and Johansen T: p62/SQSTM1 binds directly to Atg8/LC3 to facilitate degradation of ubiquitinated protein aggregates by autophagy. J Biol Chem 282: 24131-24145, 2007.

22. Kim MS, Kuehn HS, Metcalfe DD and Gilfillan AM: Activation and function of the mTORC1 pathway in mast cells. J Immunol 180: 4586-4595, 2008

23. Klionsky DJ and Emr SD: Autophagy as a regulated pathway of cellular degradation. Science 290: 1717-1721, 2000.

24. Meijer AJ and Codogno P: Regulation and role of autophagy in mammalian cells. Int J Biochem Cell Biol 36: 2445-2462, 2004.

25. Mori M, Hitora T, Nakamura O, Yamagami Y, Horie R, Nishimura $\mathrm{H}$ and Yamamoto T: Hsp90 inhibitor induces autophagy and apoptosis in osteosarcoma cells. Int J Oncol 46: 47-54, 2015. 
26. Liang X, Tang J, Liang Y, Jin R and Cai X: Suppression of autophagy by chloroquine sensitizes 5-fluorouracil-mediated cell death in gallbladder carcinoma cells. Cell Biosci 4: 10, 2014

27. Liu B, Cheng Y, Zhang B, Bian HJ and Bao JK: Polygonatum cyrtonema lectin induces apoptosis and autophagy in human melanoma A375 cells through a mitochondria-mediated ROS-p38-p53 pathway. Cancer Lett 275: 54-60, 2009.

28. Wang Q, Chen Z, Diao X and Huang S: Induction of autophagydependent apoptosis by the survivin suppressant YM155 in prostate cancer cells. Cancer Lett 302: 29-36, 2011.

29. Nakamura O, Hitora T, Yamagami Y, Mori M, Nishimura $H$, Horie R, Yamaguchi K and Yamamoto T: The combination of rapamycin and MAPK inhibitors enhances the growth inhibitory effect on Nara-H cells. Int J Mol Med 33: 1491-1497, 2014.
30. Correa RJ, Valdes YR, Peart TM, Fazio EN, Bertrand M, McGee J, Préfontaine M, Sugimoto A, DiMattia GE and Shepherd TG: Combination of AKT inhibition with autophagy blockade effectively reduces ascites-derived ovarian cancer cell viability. Carcinogenesis 35: 1951-1961, 2014.

31. Nishikawa T, Tsuno NH, Okaji Y, Shuno Y, Sasaki K, Hongo K, Sunami E, Kitayama J, Takahashi K and Nagawa H: Inhibition of autophagy potentiates sulforaphane-induced apoptosis in human colon cancer cells. Ann Surg Oncol 17: 592-602, 2010.

32. Nakamura O, Hitora T, Akisue T, Kawamoto T, Yamagami Y and Yamamoto T: Inhibition of induced autophagy increases apoptosis of Nara-H cells. Int J Oncol 39: 1545-1552, 2011. 